\title{
Numerical Simulation of the Formation of Hydrated Electron States
}

\author{
Alina Volokhova ${ }^{1, \star}$, Elena Zemlyanaya ${ }^{1}$, Viktor Lakhno $^{2}$, Ilkizar Amirkhanov ${ }^{1}$, Maxim Bashashin ${ }^{1}$, \\ Igor Puzynin ${ }^{1}$, and Taisiya Puzynina ${ }^{1}$ \\ 1 Joint Institute for Nuclear Research, 141980 Dubna, Russia; \\ ${ }^{2}$ Institute of Mathematical Problems of Biology, Russian Academy of Science, 142290 Pushchino, Russia
}

\begin{abstract}
Numerical simulation of the photoexcited electron states formation in water under the action of the ultraviolet range laser irradiation is carried out on the basis of the polaron model with a time-dependent calculation of the hydrated electron absorption band width. This framework is shown to reproduce well the experimental data on the light absorption by the hydrated electron in polarized water. Effectiveness of parallel implementation is tested on the HybriLIT cluster.
\end{abstract}

\section{Introduction}

The theoretical and experimental study of the solvated electron is motivated by its wide applicability to the theoretical explanations of complex processes in chemistry, biology and quantum physics [1-3]. For the numerical study of the hydrated electron, we start with the polaron model formulated in [4]. The numerical method and parallel computer code for solving the respective system of nonlinear partial differential equations, has been developed in [5, 6]. In [7], the results of numeral simulation of the hydrated electron formation process were shown to be in a reasonable agreement with experimental data from [8] on the absorption of the light in the polarized water. In this contribution, the model is extended to include time-dependent calculation of the hydrated electron absorption band width. Numerical simulations within the frame of the advanced model provide a noticeable improvement of agreement between experimental data and theoretical results in full range of time. Also, the results of methodical calculations demonstrating good efficiency of the parallel implementation on the basis of the partition algorithm [9] are presented.

\section{Mathematical model and numerical approach}

In the frame of the polaron model, the hydrated electron state is described by the following equations $[4,7,10]:$

$$
\begin{gathered}
i \hbar \dot{\Psi}(\vec{r}, t)=-\frac{\hbar^{2}}{2 m} \Delta \Psi(\vec{r}, t)-e \Phi(\vec{r}, t) \Psi(\vec{r}, t), \\
\Delta \Phi(\vec{r}, t)=\theta(\vec{r}, t),
\end{gathered}
$$

\footnotetext{
^e-mail: volokhova@jinr.ru
} 


$$
\frac{1}{4 \pi \omega^{2} c} \ddot{\theta}(\vec{r}, t)+\gamma \dot{\theta}(\vec{r}, t)+\frac{1}{4 \pi c} \theta(\vec{r}, t)+e|\Psi(\vec{r}, t)|^{2}=0 .
$$

where $m$ is the effective electron mass, $c=\varepsilon_{\infty}^{-1}-\varepsilon_{0}^{-1}, \varepsilon_{\infty}$ and $\varepsilon_{0}$ are the high-frequency and static constants, $e$ is the elementary charge, $\omega$ is the frequency of optical polarization medium oscillations, $\Phi$ is the potential appearing during the polarization process, $\theta$ describes the density of polarized charge induced by the electron. The relaxation (friction) $\gamma$ is determined by the coefficient related to the dielectric relaxation time $\tau$ by the expression $\gamma^{-1}=\omega^{2} \tau c$. Expanding the functions $\Psi(\vec{r}, t) \Phi(\vec{r}, t)$, and $\theta(\vec{r}, t)$ in spherical harmonics, restricting our consideration to the spherically symmetric case, and coming to dimensionless variables, we obtain the following system of spatially one-dimensional partial differential equations with respect to the radial components $\psi(r, t), \varphi(r, t)$ and $\Theta(r, t)$ of the functions $\Psi, \Phi$ and $\theta$ :

$$
\left\{\begin{array}{l}
{\left[i 2 \bar{m} \frac{\partial}{\partial t}+\frac{\partial^{2}}{\partial x^{2}}+2 \bar{m} \frac{r_{00}}{\tilde{\epsilon}} \frac{\varphi(x, t)}{x}\right] \psi(x, t)=0,} \\
\frac{\partial^{2}}{\partial x^{2}} \varphi(x, t)=\Theta(x, t), \\
{\left[\frac{\partial^{2}}{\partial t^{2}}+\bar{\gamma} \frac{\partial}{\partial t}+\bar{\omega}^{2}\right] \Theta(x, t)=-\bar{\omega}^{2} \frac{|\psi(x, t)|^{2}}{x}}
\end{array} .\right.
$$

The boundary conditions have the form:

$$
\varphi(0, t)=\varphi^{\prime}(\infty, t)=0, \psi(0, t)=\psi(\infty, t)=0, \Theta(0, t)=\Theta(\infty, t)=0 .
$$

Here, $\bar{m}=2.692, \bar{\gamma}=2.145$, and $\bar{\omega}=1$ are the dimensionless parameters of the effective mass of the hydrated electron, the relaxation (friction), and the frequency; $\tilde{\epsilon}=1.81$ is a constant coefficient; $r_{00}=164.64$ is a scaling factor. The relations between the dimension and dimensionless variables are given in $[7,10]$.

For numerical solution, the system (4) is replaced with that of difference equations on a uniform discrete mesh in the coordinate $x$ and time $t$. We employ the algorithm described in $[5,10]$ making it possible to sequentially calculate, at each time step, the solutions $\Theta(x, t), \varphi(x, t), \psi(x, t)$ at the $x$-nodes of the discrete mesh under given initial condition $\psi(x, 0)$. The MPI/C++ parallel implementation of this scheme on the basis of partition algorithm [9] has been developed in [10,11].

\section{Numerical results}

To reproduce the experimental values of the intensity of light absorption by a hydrated electron, we chose the initial condition of the wave function $\psi$ in the Gaussian form with parameter $\sigma$ which is varied to reproduce, in the computer simulation, the time dynamics of the light absorption by water.

The light absorption $I(t)$ is calculated as follows [4]:

$$
I(t)=\frac{4 \Omega^{2} \gamma_{s}^{2}}{\left(W(t)^{2}-\Omega^{2}\right)^{2}+4 \Omega^{2} \gamma_{s}^{2}},
$$

where $\gamma_{s}$ is the width of the absorption band of the hydrated electron and $\Omega$ denotes of the light frequency of a scanning laser at which the light is absorbed by the hydrated electron.

In [7], we fixed $\gamma_{s}$ to the constant value $\gamma_{s}=0.38 \mathrm{eV}$. The model provided a reasonable agreement with the experimental data except for the region of zero and small time, where a noticeable discrepancy between numerical and experimental data was observed [7, 10]. Here, $\gamma_{s}(t)$ at each step of time is calculated as follows:

$$
\gamma_{s}(t)=\sqrt{\hbar \omega k_{B} T} \sqrt{2 \ln 2} \sqrt{a(t)}, \quad a(t)=\int_{0}^{r_{\max }} d r\left[\int_{0}^{r} d r^{\prime}\left|\psi\left(r^{\prime}, 0\right)\right|^{2}-\int_{0}^{r} d r^{\prime}\left|\psi\left(r^{\prime}, t\right)\right|^{2}\right]^{2},
$$



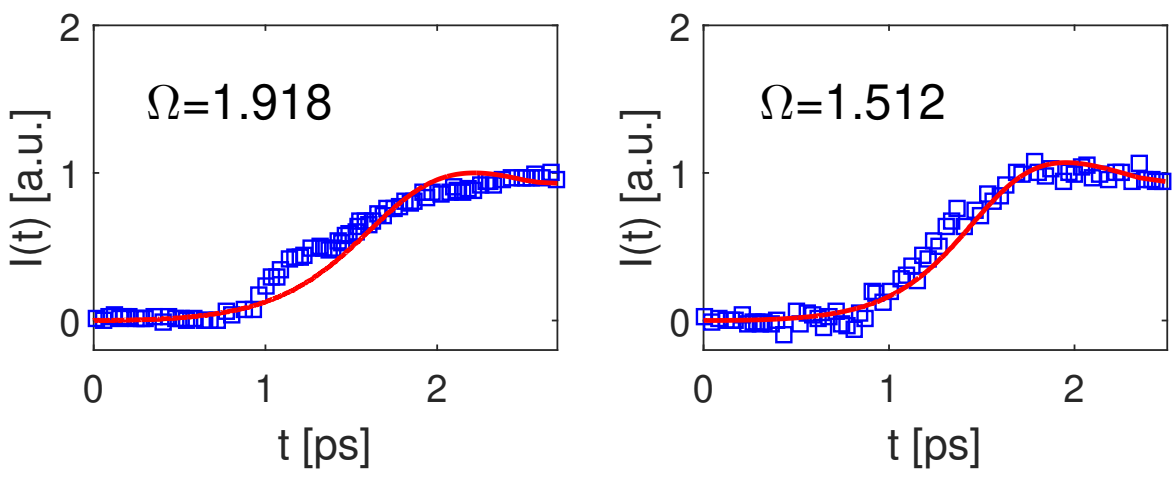

Figure 1. Numerical simulation of the light absorption by a hydrated electron for experimental data [8] derived at light frequencies of the scanning laser $\Omega=1.918 \mathrm{eV}$ (left) and $\Omega=1.512 \mathrm{eV}$ (right).
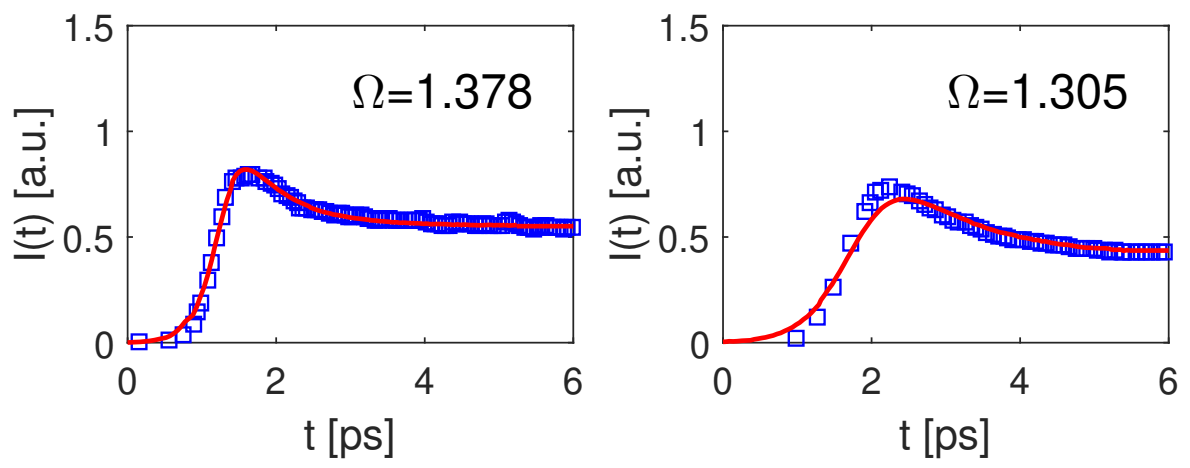

Figure 2. Same as Fig. 1 for experimental data [12] at $\Omega=1.378 \mathrm{eV}$ (left) and $\Omega=1.305 \mathrm{eV}$ (right).

where $T$ is the absolute temperature, $k_{B}$ is the Boltzmann constant. Results of the simulations are given in Fig. 1 and Fig. 2 in comparison with the experimental data from [8] and [12], respectively. Corresponding values of Gaussian parameter $\sigma$ are given in the Table 1. It is seen that the modified approach with time-dependent calculation of $\gamma_{s}$ ensures good agreement with the experimental data over all the range of the spectrum.

Table 1. Values $\Omega$ and fitted values of the Gaussian parameter $\sigma$ providing good agreement of numerical results with experimental data from $[8,12]$.

\begin{tabular}{lllll}
\hline Reference & {$[8]$} & {$[8]$} & {$[12]$} & {$[12]$} \\
$\Omega$ & 1.918 & 1.512 & 1.378 & 1.305 \\
$\sigma$ & 5.750 & 4.500 & 3.000 & 6.500 \\
\hline
\end{tabular}

Methodical calculations with different number of computing nodes have been made on the heterogenous cluster HybriLIT (Laboratory of IT, Joint Institute for Nuclear Research, Dubna, http://hybrilit.jinr.ru/en/) with $2 \cdot 10^{6}$ steps in time. The execution time versus the number $P$ of parallel computing nodes is shown in Fig. 3 (left panel) to demonstrate the effectiveness of the parallel $\mathrm{C}++$ /MPI code. It is seen that the execution time is decreasing hyperbolically while $P \leq 12$. In the left panel of Fig. 3, the maximal acceleration is given versus the number of $x$-nodes. 

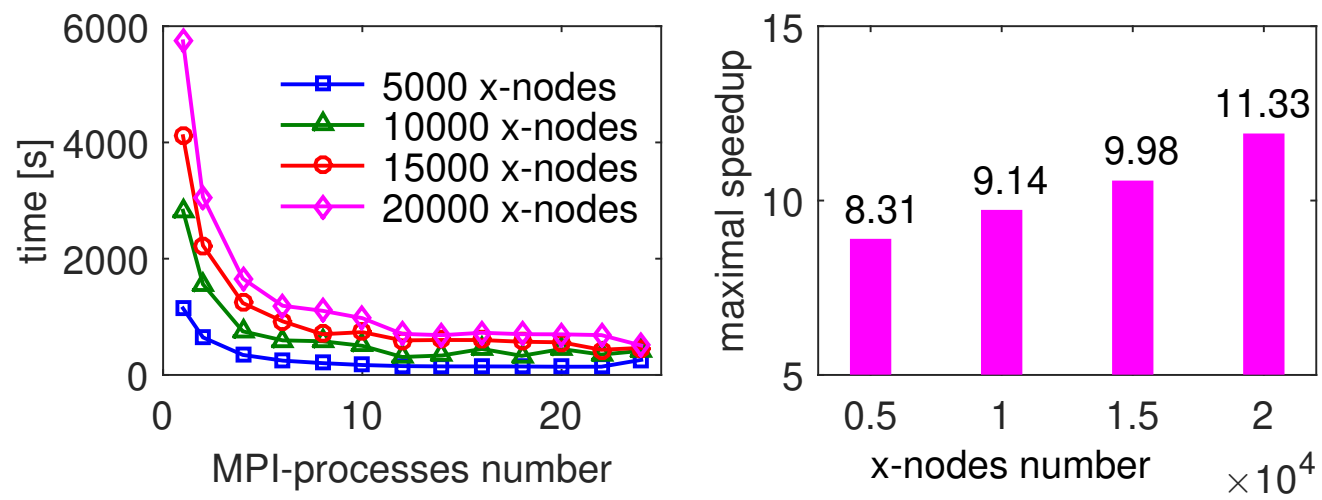

Figure 3. Left panel: dependence of the execution time on number of parallel MPI-processes for the cases of 5000, 10000, 15000, and $20000 x$-nodes of the discrete grid. Right panel: Maximal acceleration in dependence on number of $x$-nodes. Calculations on the HybriLIT cluster (Dubna).

\section{Summary}

We have found that the polaron model with time-dependent calculation of $\gamma_{s}$ provides realistic description of the light absorbtion dynamics by the photoexcited hydrated electron at light frequencies $\Omega$ of a scanning laser in the range 1.3-1.9 eV. The model can be used for further simulations and predictions of the polaron states dynamics in the aqueous medium and in other condensed media. An MPI-based parallel algorithm is shown to provide the 8-11 times acceleration in comparison with the serial calculations.

\section{Acknowledgements}

The work was supported by the RFBR (grants 17-01-00661a, 16-07-00305a).

\section{References}

[1] A.K. Pikaev, The Solvated Electron in Radiation Chemistry (World University, Jerusalim, 1970)

[2] K.R. Siefermann, Y. Liu, and E. Lugovoy, et al., Nature chemistry 2, 274-279 (2010)

[3] Y. Tang, H. Shen, K. Sekiguchi, et al., Phys. Chem. Chem. Phys. 12, 3653-3655 (2010)

[4] V.D. Lakhno, Chem. Phys. Lett. 437, 198-202 (2007)

[5] I.V. Amirkhanov, E.V. Zemlyanaya, V.D. Lakhno, et al., J. Surface Investigation 5, 1, 60-64 (2011)

[6] Volokhova A.V. Zemlyanaya E.V. Lakhno V.D., et al. Komp'yut. Issled. Modelir. (Russian) 6, 2, 253-261 (2014)

[7] V.D. Lakhno, A.V. Volokhova, E.V. Zemlyanaya, et al., J. Surface Investigation 9, 1, 75-80 (2015)

[8] F.H. Long, H. Lu, and K.B. Eisenthal, Phys. Rev. Lett. 64, 1469-1472 (1990)

[9] H.H. Wang, ACM Trans. Math. Software 7, 170-183 (1981)

[10] E.V. Zemlyanaya, A.V. Volokhova, V.D. Lakhno, et al., AIP Conf. Proc. 1684100006 (2015)

[11] A.V. Volokhova, E.V. Zemlyanaya, and V.S. Rikhvitskij, Computational Methods and Programming [Russian] 16, 281-289 (2015)

[12] R. Lian, R.A. Crowell, and I.A. Shkrob, J. Phys. Chem. A 109, 1510 (2005) 